\title{
[ 287 ]
}

follow; that, on any mufcular exertion, the heart muft have been overloaded; the difficulty of the circulation greatly increafed; and the blood detained in all the ramifications of the fanguineous fyftem. In this manner Dr. Pulteney accounts, in a fatisfactory manner, for the fymptoms under which the patient laboured, fuch as the fenfe of oppreffion upon motion; the faintnefs and difficulty of breathing; and the more or lefs conftantly-deepened tinge of the fkin, particularly that of the face and hands.

VIII. Case of a remarkably large Timour found in the Cavity of the Abdomen. By Johnua Firher, F. A. A. and M. S. - From the Memoirs of the American Academy of Arts and Sciences, Vol. I. 4to. Bofton, 1785 .

THE fubject of the following memoir was I a woman, of ftrong habit, rather fpare than grofs, and of an active difpofition. She lived in a married ftate from early life, but never had a child; was not peculiarly fubject: to any diforder, except fome florid cutaneous eruptions, till at about the ufual time of life the 


\section{[ 288 ]}

the catamenia ceafed. Soon after that periof fhe became fenfible of an unufual fulnefs in the abdomen, which continued almoft imperceptibly increafing, without any difagreeable fymptoms, till the was near fixty years old, about two years before her death. She then complained of a pain in the left hypochondriac region, which became fenfibly tumefied; the pain and diftenfion from thence increafed, and fpread over the whole abdomen.

I firft faw her between four and five months before her death; I found the abdomen very: large and tenfe, efpecially on the left fide, which was the moft painful : the veffels of her head and arms were full and turgid, while an, inanition had taken place in the lower extremities, with a variety of fymptoms arifing from an unequal diftribution of the circulating fluids and nervous influence. But the immediate caufe of her principal complaints was an inflammation, which appeared to be feated before the rectum; although fcarcely any part of the abdomen was exempt from attacks of the moft excruciating pain at intervals, yet the lower and pofterior parts were principally affected; and while a fuppuration was forming, (which had probably taken place feveral times before 


\section{[ 289 ]}

before I faw her) the pain darted a little backward and downward, and terminated in the rectum, from which a quantity of bloody pus was, at length, difcharged. Her bowels were generally conftipated, and when the fymptoms were the moft fevere, the difcharge of urine was fmall, and attended with pain.

The principal medicines that I made ufe of were, the faline draught, and Ol. Ricin. with anodynes occafionally. In the fpace of a few weeks the fymptoms of inflammation difappeared, the fecretions and excretions became free, and, by the ufe of the bark and gentle exercife, the recovered a confiderable degree of ftrength, the fymptoms arifing merely from diftention and compreffion being very tolerable. This truce lafted about two months: a fudden fuppreffion of perfpiration was followed by a return of her complaints with redoubled violence, and fome paralytic fymptoms. On the twelfth day of her relapfe the difcharged a confiderable quantity of purulent matter, intermixed with fome blood and fæces, but without any relief, and the died on the fourteenth. In her laft ftruggles, which were very violent, the difcharged (probably per vaginam) a quantity of darkifh water, refembling high-coloured urine, Vó. VII. PART III. P p amount- 


\section{[ 290 ]}

amounting, by eftimation, to at leaft two gallons. This difcharge confiderably reduced the bulk of the abdomen, efpecially of the left fide,

The day after her death I went about noon, twith the Rev. Mr. Cutler, of Ipfiwich, and Dr. Spafford, of Beverly, to open the body. We expected that it would not be buried till the day following; but, to our great difappointment, found it was to be interred the fame afternoon: at length, however, we were aliowed an hour to examine it.

On opening the abdomen, which now appeared about as large as in the laft month of pregnancy, a preternatural fubftance prefented itfelf, fituate a little more to the right fide than to the left, and occupying nearly the whole cavity. After feparating it from the contiguous parts, to all of which it firmly adhered, its general figure appeared to be that of a cone or pear; the lower part (fuppofing the body ereet) being within the pelvis, was very nearly conical; the upper part was divided by two grooves, running in a right line from its bafis, towards the apex, the one on its anterior, the other on its pofterior fide, dividing it into two lateral portions, and giving it the appearance of its having been formed by two globular bodies 


\section{[ $2 g r$ ]}

Bodies compreffed together. The pofterior groove correfponded to the projection of the fpine, to which it adhered; the anterior groove was wider, and the protuberances on each fide fomewhat larger. The bafis lay above the kidneys, and the apex nearly low enough to form a tumour in perineo. By cutting into it, it appeared to be an uniform fchirrus, of a cineritious colour, with veffels; or rather perfoxations, interfperfed, for conveying the circulating fluids. From its frtuation, connexions, and figure; it appeared to have originated in both the ovaria; the two fchirrofities in procefs of time having united, and; meeting with the leaft refiftance from below, extended into the pelvis till the cavity was completely filled. It weighed upwards of ten pounds and a quarter, averdupois weight. We made fome obfervations on the neighbouring vifcera; but, for want of time, they were unavoidably very imperfect.

The vefica urinaria was moderately diftended with urine; forming a tumour over the offa pubis, the neck being compreffed between the pubis and the fchirrus. Its whole anterior furface was firmly attached to the peritoneum, and the poiferior to the fchirrus, except a fmall portion in the middle of it, which adhered to

$$
\mathrm{Pp}_{2} \text { the }
$$




\section{[ $2 g z]$}

the uterus, where that vifcu's intervened. The ureters, for the fpace of two or three inches, feemed to lie loofe, and contained a fmall quantity of urine; their coats being thin and confiderably diftended. They then entered upon the fchirrus, one on each fide, involved in a membrane hereafter mentioned, running obliquely forward and downward till they entered the bladder. After they were connected to the fchirrus, their appearance was the reverfe of the former : to enable them to take the circuit, they had been greatly extended, and their diameters were exceedingly fmall. The uterus was extended in a right line upon the fchirrus from its bafis (to which the fundus uteri adhered) through the anterior groove, almoft to its apex, continuing about an inch and a quarter wide, and firmly attached through its whole length. Its coats appeared in a natural ftate, except that they were thinner by reafon of theit extenfion.

The appearance of the tuba fallopianz was fo unnatural, that they were fcarcely diftinguifhable. We found a membranous covering clofely embracing the bafis, the anterior, and lateral fides of the fchirrus, extending downward nearly to the pelvis; and by its upper 


\section{[ 293 ]}

and lateral edges adhering to the fchirrus, it feemed to arife from the uterus, which was fituated in the middle of it, and its external membrane was a continuation of the external membrane of the uterus. Through this expanfion on the bafis of the fchirrus were feveral fmooth, but irregular, apertures, of about two inches circumference, through which the bafis of the fchirrus appeared; it was here about a quarter of an inch thick, its fibres flefhy and variounly convolved; the lower part of it was much thinner, and purely membranous. That part of this tegument which was fpread upon and near the bafis appeared to be formed by a diftention of the fallopian tubes included in the peritoneum, or common membrane of the abdomen, and the remainder of it by an expanfion of thofe duplicatures of the fame membrane, ufually termed ligamenta lata.

On the pofterior fide the fchirrus was attached to the aorta, vena cava, \&c.; to the rectum, which was protruded to the right fide of the fpine, it adhered for the fpace of feven or eight inches: near the bafis the adhefron was ftrong; but a little lower the connecting membrane contained a quantity of grumous blood, 


\section{[ 294$]$}

appeared putrid, and in fome places was ded Aroyed.

We wifhed to have afcertained the fource of the water which the patient difcharged at the time of her death: by the appearance of the vifcera there had been none diffured in the cavity of the abdomen; and, from the circumftances above mentioned, it muft have been contained in the left hypochondriun. In removing the fchirrus we found a membrane (a fac to appearance) adhering to it, which we cut through, and fuppofed to be an empty hydropic cyft; but the fchirrus at that inftant engroffed our attention, and people foon collect. ing to attend the funeral, all farther examination was prevented.

We obferved nothing extraordinary in any of the other vifcera : the omentum, as is ufual in tabid cafes, was nearly wafted, and a number of the mefenteric glands were enlarged and indurated.

IX. A remarkable Cafe of a Guin-fiot Wound. Communicated in a Letter from Barnabas Binney, Horpital Pbyfician, and Surgeon its the 